\title{
A Mode-matching Approach for the Analysis and Design of Substrate-integrated Waveguide Components
}

\author{
Jens Bornemann, ${ }^{1, *}$ Farzaneh Taringou ${ }^{1}$ and \\ Zamzam Kordiboroujeni ${ }^{1}$ \\ ${ }^{1}$ Department of Electrical and Computer Engineering, \\ University of Victoria, Canada
}

\begin{abstract}
A mode-matching approach is presented that allows a fast and accurate analysis of substrate-integrated wave-guide components with rectangular/square via holes. Models for several discontinuities are discussed which include microstrip as well as all-dielectric wave-guide feeds. The numerical technique is verified by comparison with commercially available field solvers. An example of a fourpole dual-mode filter in substrate-integrated wave-guide technology illustrates the capabilities of the approach.
\end{abstract}

Keywords. Mode-matching techniques, substrateintegrated wave-guide technology, numerical modeling, computer-aided design.

PACS $^{\circledR}$ (2010). 84.40.Az, 84.40.DC, 85.40.Bh.

\section{Introduction}

Numerical modelling approaches based on modal expansion techniques have long been known as reliable and computationally efficient analysis tools for waveguide-based transmission media [1,2]. Especially with a reduced mode set, such as in H-plane waveguide circuits [3], E-plane [4] or iris-type configurations [5], the classical ModeMatching Technique (MMT) presents a powerful method for the computer-aided analysis and design of air-filled or dielectric-slab-loaded waveguide components [6,7].

More recently, substrate-integrated circuits emerged [8]. Especially the Substrate-Integrated Waveguide (SIW) presents a now widely accepted circuit compromise in the lower millimetre-wave regime where microstrip components are increasingly lossy and waveguides too bulky and too expensive. Thus a large variety of SIW circuits has appeared in the literature covering frequency ranges from

Corresponding author: Jens Bornemann, Department of Electrical and Computer Engineering, University of Victoria, Victoria, B.C., V8W 3P6, Canada; E-mail: j.bornemann@ieee.org.

Received: June 20, 2011.
$5 \mathrm{GHz}$ [9], over 30 and $60 \mathrm{GHz}[10,11]$, to above $100 \mathrm{GHz}$ $[12,13]$.

SIW circuit designs are usually carried out within commercial field solvers such as CST Microwave Studio or Ansoft HFSS. They provide accurate responses for the analysis of a given SIW structure within reasonable time frames. In optimization mode, however, the numerical effort of such packages is tremendous and time-consuming, especially for frequency-sensitive structures such as bandpass filters. Thus other techniques, which are more geared towards waveguide-type modeling, are in demand. So far, the MMT is used for dispersion analysis [14], and the Boundary Integral-Resonant Mode Expansion (BI-RME) method for equivalent-circuit extraction [15]. Although the SIW is an H-plane circuit and would lend itself straightforwardly to MMT analysis and design, the circular via holes applied in common integrated-circuit fabrication techniques complicate the MMT formulation and thus increase computational complexity. However, with the emergence of new fabrication techniques, especially laser drilling of via holes, more arbitrary via shapes become feasible [12], and rectangular/square via shapes have been implemented in [13].

Therefore, this paper presents a mode-matching approach for the analysis and design of SIW components with square via holes. The numerical procedure follows fundamental MMT principles in [16] and includes SIW feeding by microstrip transformers [17] or all-dielectric waveguide [18].

\section{Theory}

Figure 1 shows a SIW consisting of ten pairs of equally spaced square via holes. It is fed by a microstrip line and a short microstrip transformer [19] at the input and an alldielectric waveguide port at the output.

In order to model such a circuit, we have to consider several different discontinuities. First, a microstrip-tomicrostrip discontinuity to analyse the transformer which will be represented by staircasing in the MMT procedure; secondly, a microstrip-to-dielectric waveguide discontinuity to capture the step from the microstrip line to the dielectric waveguide containing the via holes; thirdly, the discontinuity from the dielectric waveguide to an $\mathrm{N}$-furcated waveguide, representing a via-holed section, and back to the dielectric waveguide; and fourthly, a discontinuity between two dielectric waveguides to model the output port. 


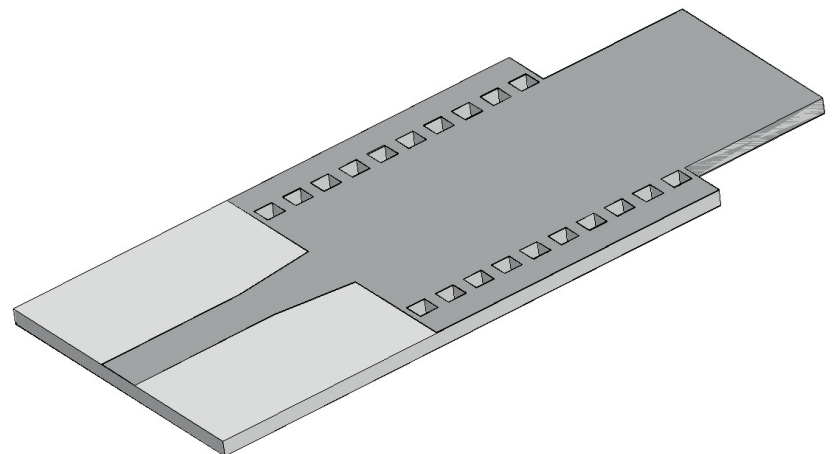

Figure 1. Substrate-integrated waveguide with microstrip input and all-dielectric waveguide output ports.

As pointed out in Section 1, the MMT is especially efficient if a reduced set of modes can be used. Due to the small thickness of the substrate, only $\mathrm{TE}_{m 0}$ modes are considered in all-dielectric waveguides. In all microstrip sections, the quasi TEM as well as all applicable $\mathrm{TE}_{m 0}$ modes are considered. Since the TEM mode is the lowest possible TM mode, the overall electromagnetic field can be calculated from two axial vector potential components, $A_{e z}$ and $A_{h z}$, as

$$
\begin{aligned}
\vec{E} & =\frac{1}{j \omega \varepsilon} \nabla \times \nabla \times\left(A_{e z} \vec{e}_{z}\right)-\nabla \times\left(A_{h z} \vec{e}_{z}\right), \\
\vec{H} & =\frac{1}{j \omega \mu} \nabla \times \nabla \times\left(A_{h z} \vec{e}_{z}\right)+\nabla \times\left(A_{e z} \vec{e}_{z}\right),
\end{aligned}
$$

where the TE modes are represented by

$$
\begin{aligned}
A_{h z}^{v}=\sum_{m} \sqrt{Z_{h m}^{v}} \underbrace{\frac{a_{v}}{m \pi} \sqrt{\frac{2}{a_{v} b}} \cos \left\{\frac{m \pi}{a_{v}}\left(x-x_{v}\right)\right\}}_{T_{h m}^{v}} \\
\cdot\left[F_{m}^{v} \exp \left(-j k_{z m}^{v} z\right)+B_{m}^{v} \exp \left(+j k_{z m}^{v} z\right)\right]
\end{aligned}
$$

and the TEM mode by

$$
A_{e z}^{v}=\sqrt{Y_{\ell}^{v}} \underbrace{\frac{y}{\sqrt{a_{v} b}}}_{T_{e \ell}^{v}(y)}\left[F_{\ell}^{v} \exp \left(-j k_{z \ell}^{v} z\right)-B_{\ell}^{v} \exp \left(+j k_{z \ell}^{v} z\right)\right]
$$

In (2) and (3), $v$ identifies the section of equivalent waveguide width $a_{v}$ with $x_{v}$ as its lower coordinate, $b$ is the substrate height, $F$ and $B$ are the forward and backward travelling wave amplitudes, respectively, $k_{z}$ 's are the propagation constants

$$
k_{z i}^{v}=\sqrt{\left(\frac{\omega}{c}\right)^{2} \varepsilon_{\mathrm{reff}}-\left(\frac{i \pi}{a_{v}}\right)^{2}},
$$

where $i=0$ for the TEM mode, $c$ is the speed of light in free space, and the wave impedances are given by

$$
Z_{h i}^{v}=\frac{\omega \mu_{0}}{k_{z i}^{v}}=\frac{1}{Y_{h i}^{v}}, \quad Y_{\ell}^{v}=\frac{\omega \varepsilon_{0} \varepsilon_{\mathrm{reff}}}{k_{z \ell}^{v}}=\frac{1}{Z_{\ell}^{v}} .
$$

Note that the vector potential components in (2) an (3) are power normalized so that the coupling integrals, e.g., for the case shown in Figure 2 can be computed from

$$
\begin{aligned}
\left(J_{l l}\right)_{0,0} & =\int_{A_{1}}\left(\nabla T_{e \ell}^{\mathrm{I}}\right) \cdot\left(\nabla T_{e \ell}^{\mathrm{II}}\right) d a \\
\left(J_{l h}\right)_{0, k} & =\int_{A_{1}}\left(\nabla T_{e \ell}^{\mathrm{I}}\right) \cdot\left(\nabla T_{h k}^{\mathrm{II}} \times \vec{e}_{z}\right) d a \\
\left(J_{h l}\right)_{m, 0} & =\int_{A_{1}}\left(\nabla T_{h m}^{\mathrm{I}} \times \vec{e}_{z}\right) \cdot\left(\nabla T_{e \ell}^{\mathrm{II}}\right) d a, \\
\left(J_{h h}\right)_{m, k} & =\int_{A_{1}}\left(\nabla T_{h m}^{\mathrm{I}}\right) \cdot\left(\nabla T_{h k}^{\mathrm{II}}\right) d a,
\end{aligned}
$$

where the TEM-to-TEM coupling occupies the upper left matrix element in $\mathbf{J}$.

Once the coupling integrals $\mathbf{J}$ are obtained, the coupling matrix $\mathbf{M}$ follows from

$$
\mathbf{M}=\operatorname{Diag}\left\{\sqrt{Y_{\ell m}^{\mathrm{I}}}\right\}(\mathbf{J}) \operatorname{Diag}\left\{\sqrt{Z_{\ell k}^{\mathrm{II}}}\right\} .
$$

This technique will now be applied to the individual discontinuities involved in Figure 1. For individual combinations of modal scattering matrices and adding lengths of certain sections, the reader is referred to [16].

\subsection{Microstrip-to-microstrip Discontinuity}

Figure 2 shows the discontinuity and the boundary conditions involved. Note that widths $a_{v}$ represent the equivalent and frequency-dependent waveguide widths of the actual microstrip lines with finite conductor thickness and frequency-dependent permittivity. Moreover, this discontinuity includes models to shift the reference plane due to fringing fields. For details on microstrip transmission line parameters, the reader is referred to [20].

For this case, the specific coupling integrals according to (2), (3) and (6) are

$$
\begin{aligned}
&\left(J_{l l}\right)_{0,0}=\sqrt{\frac{a_{1}}{a_{2}}}, \quad\left(J_{h l}\right)_{m, 0}=0 \\
&\left(J_{l h}\right)_{0, k}=\frac{-\sqrt{2}}{\sqrt{a_{1} a_{2}}} \int_{-a_{1} / 2}^{+a_{1} / 2} \cos \left\{\frac{k \pi}{a_{2}}\left(x+\frac{a_{2}}{2}\right)\right\} d x \\
&\left(J_{h, h}\right)_{m, k}=\frac{2}{\sqrt{a_{1} a_{2}}} \int_{-a_{1} / 2}^{+a_{1} / 2} \cos \left\{\frac{m \pi}{a_{1}}\left(x+\frac{a_{1}}{2}\right)\right\} \\
& \cdot \cos \left\{\frac{k \pi}{a_{2}}\left(x+\frac{a_{2}}{2}\right)\right\} d x
\end{aligned}
$$




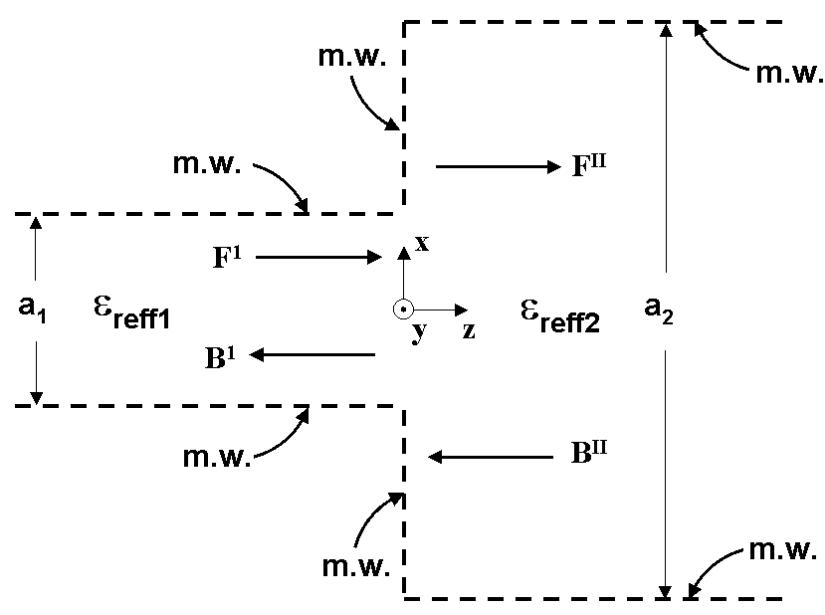

Figure 2. Microstrip-to-microstrip discontinuity.

Note that $T_{h}$ in (2) depends only on $x$, whereas $T_{e}$ in (3) depends only on $y$.

The modal scattering matrix for Figure 2 is defined as

$$
\left[\begin{array}{c}
\mathbf{B}^{\mathrm{I}} \\
\mathbf{F}^{\mathrm{II}}
\end{array}\right]=\left[\begin{array}{ll}
\mathbf{S}_{11} & \mathbf{S}_{12} \\
\mathbf{S}_{21} & \mathbf{S}_{22}
\end{array}\right]\left[\begin{array}{c}
\mathbf{F}^{\mathrm{I}} \\
\mathbf{B}^{\mathrm{II}}
\end{array}\right]
$$

and once the coupling matrix $\mathbf{M}$ is computed from (7) and (8), the scattering submatrices follow as

$$
\begin{aligned}
\mathbf{S}_{11} & \left.=\left[\mathbf{M} \mathbf{M}^{T}+\mathbf{U}\right]^{-1}\right]\left[\mathbf{M} \mathbf{M}^{T}-\mathbf{U}\right], \\
\mathbf{S}_{12} & =2\left[\mathbf{M} \mathbf{M}^{T}+\mathbf{U}\right]^{-1} \mathbf{M}=\mathbf{S}_{21}^{T}, \\
\mathbf{S}_{21} & =\mathbf{M}^{T}\left[\mathbf{U}-S_{11}\right]=\mathbf{S}_{12}^{T}, \\
\mathbf{S}_{22} & =\mathbf{U}-\mathbf{M}^{T} \mathbf{S}_{12},
\end{aligned}
$$

where $\mathbf{U}$ denotes the unit matrix.

\subsection{Microstrip-to-dielectric-waveguide Discontinuity}

Figure 3 shows the boundary conditions of the microstrip to dielectric waveguide discontinuity. Width $a_{1}$ is the equivalent waveguide width of the actual microstrip line [20].

For this case the coupling integrals are

$$
\begin{aligned}
& \left(J_{l h}\right)_{0, k}=\frac{\sqrt{2}}{\sqrt{a_{1} a_{2}}} \int_{-a_{1} / 2}^{+a_{1} / 2} \sin \left\{\frac{k \pi}{a_{2}}\left(x+\frac{a_{2}}{2}\right)\right\} d x \\
& \left(J_{h, h}\right)_{m, k}=\frac{-2}{\sqrt{a_{1} a_{2}}} \int_{-a_{1} / 2}^{+a_{1} / 2} \cos \left\{\frac{m \pi}{a_{1}}\left(x+\frac{a_{1}}{2}\right)\right\} \\
& \cdot \sin \left\{\frac{k \pi}{a_{2}}\left(x+\frac{a_{2}}{2}\right)\right\} d x
\end{aligned}
$$

and the remaining procedure follows (7) and (10) to determine the modal scattering matrix. Note that the alldielectric waveguide supports no TEM mode; thus only two different coupling integrals have to be considered compared to four in (8).

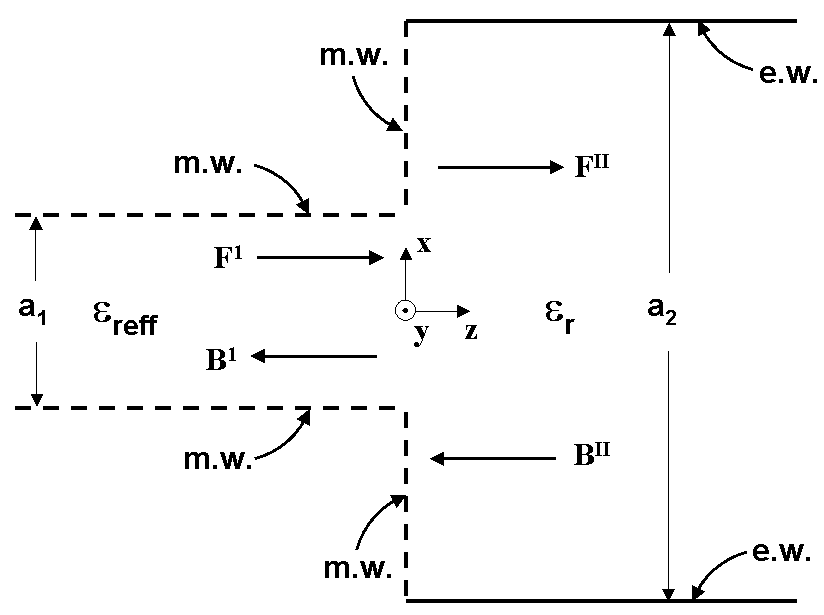

Figure 3. Microstrip-to-all-dielectric-waveguide discontinuity.

\subsection{Discontinuity Between All-dielectric Waveguides}

Figure 4 shows the boundary conditions of a discontinuity between two all-dielectric waveguides as required, e.g., at the output of the circuit in Figure 1. Since the practical realization of this circuit would be on a single rectangular substrate, the interface between the two waveguides is selected as a magnetic wall as in the two previous discontinuities involving microstrip lines. The coupling integrals for this case are simply those of the $\mathrm{TE}_{m 0}$ modes on both sides of the discontinuity

$$
\begin{aligned}
\left(J_{h, h}\right)_{m, k}=\frac{2}{\sqrt{a_{1} a_{2}}} \int_{-a_{1} / 2}^{+a_{1} / 2} \sin \left\{\frac{m \pi}{a_{1}}\left(x+\frac{a_{1}}{2}\right)\right\} \\
\cdot \sin \left\{\frac{k \pi}{a_{2}}\left(x+\frac{a_{2}}{2}\right)\right\} d x
\end{aligned}
$$

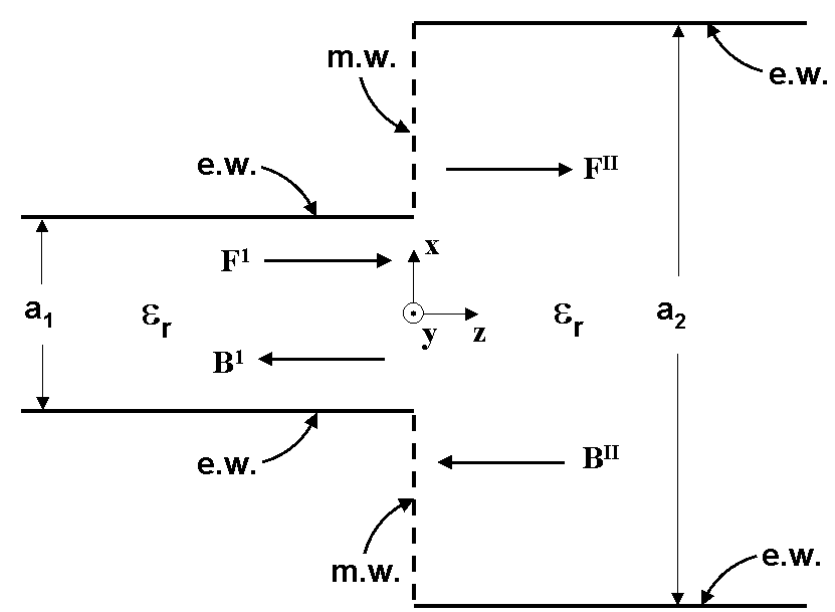

Figure 4. Discontinuity between two all-dielctric waveguides. 
because neither sections support TEM-mode propagation. The modal scattering matrix is again computed with (7) and (10).

\subsection{Via Holes Within All-dielectric Waveguide}

This discontinuity is different from the previous ones as the dielectric waveguide with $N-1$ via holes represents an $N$ furcated waveguide according to Figure 5. Note that the length of the via holes is exaggerated in order to fit in the related wave amplitudes.

Coupling integrals for this discontinuity involve coupling from the dielectric waveguide (section 0) to each of the $N$-furcated waveguides (sections $n$ ). Thus $\mathrm{N}$ coupling integrals are obtained.

$$
\begin{aligned}
\left(J_{h h}^{n}\right)_{m k}=\frac{2}{\sqrt{a_{0} a_{n}}} \int_{x_{n}}^{x_{n}+a_{n}} & \sin \left\{\frac{m \pi}{a_{0}}\left(x+\frac{a_{0}}{2}\right)\right\} \\
\cdot & \sin \left\{\frac{k \pi}{a_{n}}\left(x-x_{n}\right)\right\} d x .
\end{aligned}
$$

Applying (7) to each of the $N$ coupling integrals, the electric field matrix equation, e.g. for $N=3$, can be arranged as follows

$$
\left[\mathbf{F}^{0}+\mathbf{B}^{0}\right]=\underbrace{\left[\begin{array}{lll}
\mathbf{M}^{1} \mathbf{M}^{2} \mathbf{M}^{3}
\end{array}\right]}_{\mathbf{M}}\left[\begin{array}{l}
\mathbf{F}^{1}+\mathbf{B}^{1} \\
\mathbf{F}^{2}+\mathbf{B}^{2} \\
\mathbf{F}^{3}+\mathbf{B}^{3}
\end{array}\right],
$$

from which the scattering matrix of the discontinuity at $z=0$ and $z=L$ can be obtained according to (10). Considering the diagonal matrix between the two discontinuities, which is comprised of individual matrices

$$
D^{n}=\operatorname{Diag}\left\{\exp \left(-j k_{z k}^{n} L\right)\right\},
$$

the overall modal scattering matrix of a via-hole section is computed according to [16].

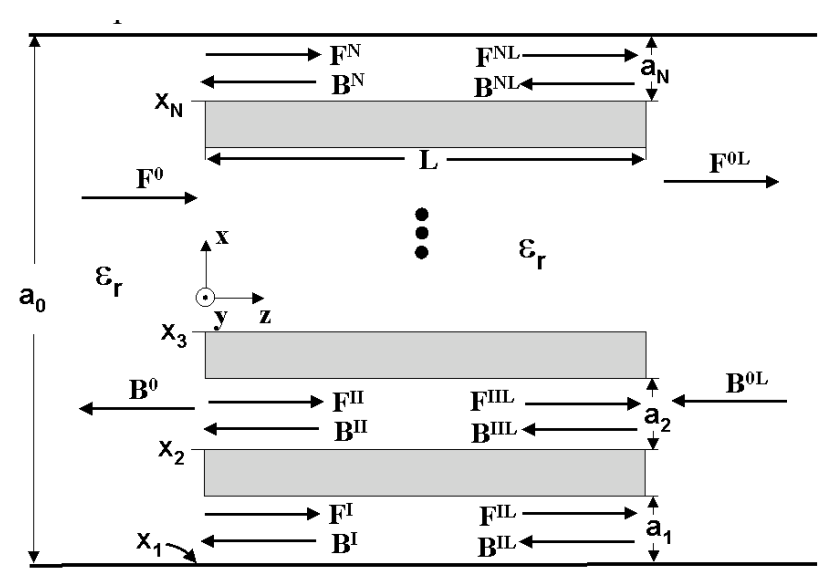

Figure 5. Discontinuities between all-dielectric waveguide and $N$-furcated waveguide formed by $N-1$ via holes.
This completes the presentation of individual discontinuities. The only issue that has been left out is a possible off-centre connection between two sections. Such an offset is easily incorporated as a shift in the sin and cos functions involved in the coupling integrals.

\section{Results}

Figure 6 shows an analysis of 10 equally spaced viahole pairs with microstrip input and all-dielectric waveguide output according to Figure 1. The substrate is chosen as RT/duroid 5880 with $\varepsilon_{\mathrm{r}}=2.2$, substrate height $b=508 \mu \mathrm{m}$ and metallization thickness $t=17 \mu \mathrm{m}$. In the mode-matching approach, the microstrip transformer at the input is approximated by five individual sections, whereas in CST and HFSS, it is modelled as shown. Very good agreement is observed between this approach (MMT), HFSS and CST, with the MMT minimum located between those of CST and HFSS.

A four-pole dual-mode filter in SIW technology is shown in Figure 7(a). The original waveguide design is from [21] and has been redesigned in all-dielectric waveguide for the $20 \mathrm{GHz}$ frequency range and transferred to SIW technology using RT/duroid 6002 substrate. The SIW filter is then analysed and fine-optimized using the method presented here. Figure 7(b) shows a direct comparison between this method (MMT) and the resonant-mode field solver in CST. Excellent agreement is observed but the MMT code is faster than CST by a factor of seven. An analysis including all dielectric and metallic losses (not shown here) reveals a midband insertion loss of $0.93 \mathrm{~dB}$ which, according to approximations in [22], determines the unloaded $\mathrm{Q}$ factor to about 550.

The same filter, but with microstrip ports, is shown in Figure 8(a). Figure 8(b) compares the results obtained with

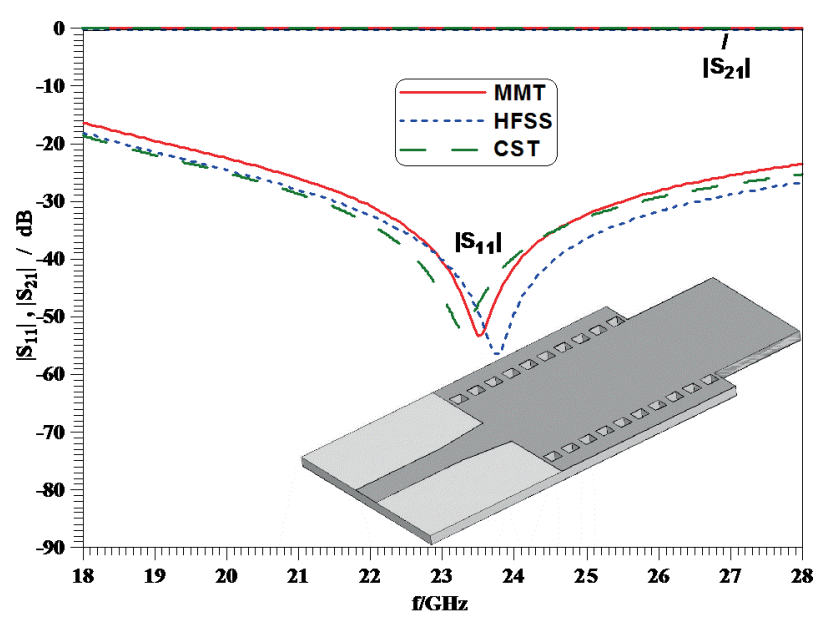

Figure 6. Comparison between results obtained with this method (MMT), HFSS and CST at the example of Figure 1. 
(a)

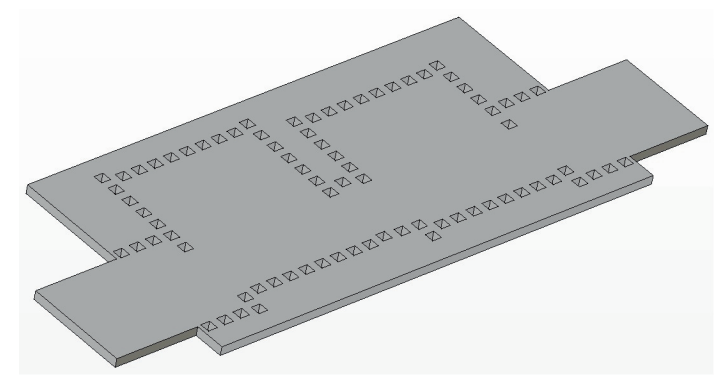

(b)

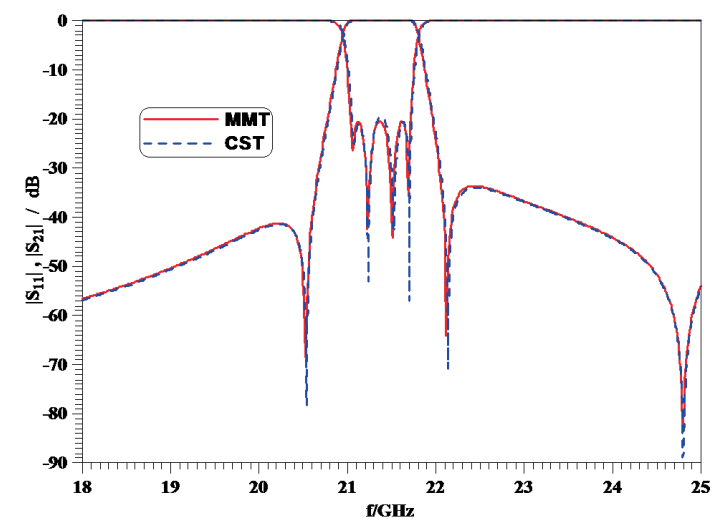

Figure 7. Four-pole dual-mode filter in SIW technology with all-dielectric waveguide ports (a) and performance comparison between this method (MMT) and CST's resonant-mode analysis (b).

(a)

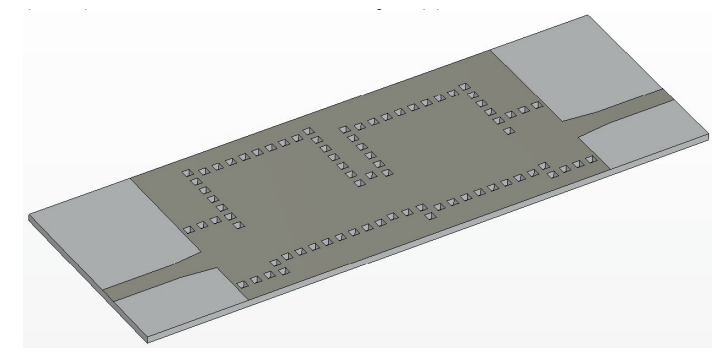

(b)

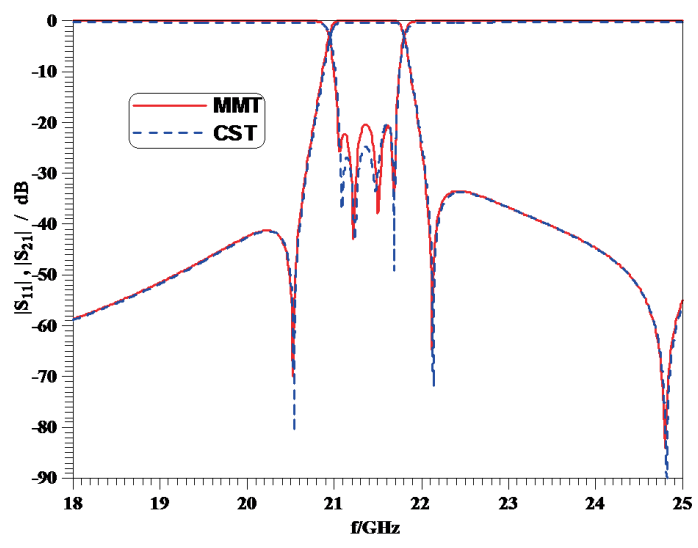

Figure 8. Four-pole dual-mode filter in SIW technology with microstrip ports (a) and performance comparison between this method (MMT) and CST's frequency domain solver (b). this method (MMT) and the frequency domain solver of CST. Some differences are observed in the return loss performance. However, the discrepancies are small and occur below the $-20 \mathrm{~dB}$ level. Therefore, the modelling procedure involving microstrip ports in MMT is verified by the CST results.

\section{Conclusions}

Substrate-integrated waveguide components with rectangular/square via holes are effectively and accurately modelled by a mode-matching approach. Two different feeds are considered, all-dielectric waveguide feeds and microstrip transformers, which allow the design to be carried out for integration with other SIW circuits or as a stand-alone component. This is demonstrated for a four-pole dual-mode SIW filter example. The results obtained with the modematching approach are verified by comparison with data of commercially available field solvers.

\section{Acknowledgments}

The authors wish to acknowledge support for this work from the Natural Sciences and Engineering Research Council of Canada.

\section{References}

[1] R. Vahldieck, Accurate hybrid-mode finline configurations including analysis of various multilayered dielectrics, finite metallization thickness, and substrate holding grooves, IEEE Trans. Microwave Theory and Tech. 32 (1984), no. 11, pp. 1454-1460.

[2] R. Vahldieck and J. Bornemann, A modified mode-matching technique and its application to a class of quasi-planar transmission lines, IEEE Trans. Microwave Theory and Tech. 33 (1985), no. 10, pp. 916-926.

[3] R. Vahldieck, J. Bornemann, F. Arndt and D. Grauerholz, Optimized E-plane metal insert filters for millimeter-wave applications, IEEE Trans. Microwave Theory and Tech. 31 (1983), no. 1, pp. 65-69.

[4] S. Amari, J. Bornemann and R. Vahldieck, On the acceleration of the coupled-integral-equations technique and its application to multistub E-plane discontinuities, J. Electro. Waves Applic. 13 (1999), no. 2, pp. 539-554.

[5] J. Bornemann and R. Vahldieck, Characterization of a class of waveguide discontinuities using a modified $\mathrm{TE}_{m n}^{x}$ mode approach, IEEE Trans. Microwave Theory and Tech. 38 (1990), no. 12, pp. 1816-1822.

[6] R. Vahldieck and W. J. R Hoefer, Finline and metal insert filters with improved passband separation and increased stopband attenuation, IEEE Trans. Microwave Theory and Tech. 33 (1985), no. 12, pp. 1333-1339.

[7] R. Vahldieck, J. Bornemann, F. Arndt and W. J. R. Hoefer, $\mathrm{TE}_{10}$-mode filters formed by multilayered dielectric slabs in waveguides below cutoff, Eur. Microwave Conf., Dublin, Ireland, Sep. 1986, pp. 275-280. 
[8] K. Wu, State-of-the-art and future perspective of substrate integrated circuits (SICs), in Workshop Notes: Substrate Integrated Circuits, IEEE MTT-S Int. Microwave Symp., Anaheim, USA, May 2010.

[9] Y. D. Dong, T. Yang and T. Itoh, Substrate integrated waveguide loaded by complementary split-ring resonators and its applications to miniaturized waveguide filters, IEEE Trans. Microwave Theory Tech. 57 (2009), no. 9, pp. 2211-2223.

[10] Q. Lai, C. Fumeaux, W. Hong and R. Vahldieck, Characterization of the propagation properties of the half-mode substrate integrated waveguide, IEEE Trans. Microwave Theory Tech. 57 (2009), no. 8, pp. 1996-2004.

[11] Q. Lai, C. Fumeaux, W. Hong and R. Vahldieck, $60 \mathrm{GHz}$ aperture-coupled dielectric resonator antennas fed by a halfmode substrate integrated waveguide, IEEE Trans. Antennas Propagat. 58 (2010), no. 6, pp. 1856-1864.

[12] E. Moldovan, R. G. Bosisio, and K. Wu, W-band multiport substrate-integrated waveguide circuits, IEEE Trans. Microwave Theory Tech. 54 (2006), no. 2, pp. 625-632.

[13] D. Dousset, K. Wu and S. Claude, Millimetre-wave broadband transition of substrate-integrated waveguide to rectangular waveguide, Electron. Lett. 46 (2010), no. 24, pp. 1610 1611.

[14] M. Salehi and E. Mehrshahi, A closed-form formula for dispersion characteristics of fundamental SIW mode, IEEE Microwave Wireless Comp. Lett. 21 (2011), no. 1, pp. 4-6.
[15] M. Bozzio and L. Perregrini, Full-wave analysis and equivalent-circuit modeling of SIW components, Workshop Notes: Substrate Integrated Circuits, IEEE MTT-S int. Microwave Symp., Anaheim, USA, May 2010.

[16] J. Uher, J. Bornemann and U. Rosenberg, Waveguide Components for Antenna Feed Systems. Theory and CAD. Artech House, Norwood, 1993.

[17] Y. Cassivi, D. Deslandes and K. Wu, Substrate integrated waveguide directional couplers, Asia-Pacific Microwave Conf., Kyoto, Japan, Nov. 2002, FR1B-3, 4 p.

[18] V. A. Labay and J. Bornemann, E-plane directional couplers in substrate-integrated waveguide technology, Asia-Pacific Microwave Conf., Hong Kong, Dec. 2008, A1-75, 4 p.

[19] D. Deslandes, Design equations for tapered microstrip-tosubstrate integrated waveguide transitions, IEEE MTT-S Int. Microwave Symp., Anaheim, USA, May 2010. pp. 704-704.

[20] R. K. Hoffmann, Handbook of Microwave Integrated Circuits, Artech House, Boston, 1987.

[21] M. Guglielmi, F. Montauti, L. Perregrini and P. Arcioni, Implementing transmission zeros in inductive-window bandpass filters, IEEE Trans. Microwave Theory and Tech. 43 (1995), no. 8, pp. 1911-1915.

[22] G. L. Matthaei, L. Young and E. M. T. Jones, Microwave Filters, Impedance Matching Networks and Coupling Structures, Artech House, Dedham: 1980. 